\title{
Influência da ascite na avaliação da função pulmonar em portadores de hipertensão portal*
}

\author{
Influence of ascites in the pulmonary function of patients with \\ portal hypertension.
}



Introdução: A oxigenação inadequada nos pacientes com hipertensão portal pode ser secundária a alterações na mecânica respiratória, determinadas pela presença da ascite.

Objetivo: Avaliar a função pulmonar de doentes com hipertensão portal antes e após redução do volume da ascite.

Métdodo: Quinze doentes com hipertensão portal e ascite foram submetidos a provas de função pulmonar constituindo-se de espirometria e gasometria arterial, antes e após redução do volume da ascite. Os parâmetros analisados foram: capacidade vital forçada (CVF); volume expiratório no primeiro segundo $\left(\mathrm{VEF}_{1}\right)$; fluxo expiratório entre 25 e 75\% da CVF (FEF 25-75\% ); volume de reserva expiratória (VRE); relação $\mathrm{VEF}_{1}$ / CVF; pressão arterial de oxigênio $\left(\mathrm{PaO}_{2}\right)$, pressão arterial de dióxido de carbono $\left(\mathrm{PaCO}_{2}\right)$ e saturação arterial de oxigênio $\left(\mathrm{SaO}_{2}\right)$.

Resultados: Houve melhora significativa dos volumes pulmonares analisados após a diminuição da ascite com 0 tratamento diurético associado ou não à paracentese.

Conclusão: Concluímos que nos doentes com hipertensão portal e ascite, há diminuição dos volumes pulmonares em relação aos valores preditos, com melhora significativa após diminuição da ascite. Do mesmo modo, observamos aumento na $\mathrm{PaO}_{2}$ e na $\mathrm{SaO}_{2}$.
Background: Oxygen deficiency in patients with portal hypertension may be secondary to changes in respiratory mechanics due to ascites.

Objectives: Evaluate pulmonary function in patients with portal hypertension before and after reduction of the ascites.

Method: Fifteen patients with portal hypertension and ascites were submitted to pulmonary function tests, comprising spirometry and arterial blood gas determination, before and after reduction of ascites. The analysed parameters were: forced vital capacity (FVC); forced expiratory volume in one second $\left(\mathrm{FEV}_{1}\right)$; forced expiratory flow between 25-75\% of the forced vital capacity (FEF 25-75\%); expiratory reserve volume (ERV); $\mathrm{FEV}_{1} / \mathrm{CVF}$; arterial oxygen pressure $\left(\mathrm{PaO}_{2}\right)$; arterial carbon-dioxide pressure $\left(\mathrm{PaCO}_{2}\right)$ and arterial oxygen saturation $\left(\mathrm{SaO}_{2}\right)$.

Results: There was remarkable improvement in pulmonary volumes after decrease of ascites by treatment with diuretics associated or not to paracentesis.

Conclusion: We concluded, that in patients with porta hypertension and ascites, there is a decrease of pulmonary volumes compared to predicted values, with significant improvement after decrease of ascites. Similarly, an increase of the arterial oxygen pressure and of the arterial oxygen saturation was perceived.

(J Bras Pneumol 2004; 30(1) 14-9

Descritores: Ascite/terapia. Hipertensão portal. Testes de função respiratória.
Key words: Ascite/therapy. Hypertension, portal. Respiratory function tests.
Siglas e abreviaturas utilizadas neste trabalho CPT: Capacidade Pulmonar Total

CRF: Capacidade Residual Funcional

CVF: Capacidade Vital Forçada

DVO: Distúrbio Ventilatório Obstrutivo

DVR: Distúrbio Ventilatório Restritivo

FEF 25-75: : Fluxo Expiratório Forçado entre 25 e 75\% da Capacidade Vital Forçada ISCMSP: Irmandade da Santa Casa de Misericórdia de São Paulo

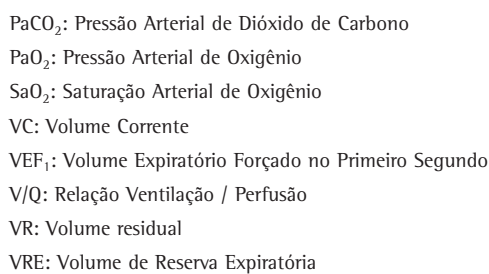

* Trabalho realizado no Serviço de Clínica Médica do Hospital São Luiz Gonzaga, na Clínica de Gastroenterologia e no Laboratório de Prova de Função Pulmonar da Irmandade da Santa Casa de Misericórdia de São Paulo. Endereço para correspondência: Ângela Maria Stiefano Nitrini. Av. Michel Ouchana, 94 - Internação Medicina - Jaçanã - S.Paulo - CEP 02273000. Tel:(11) 6982 5289 (11) 995352 67; FAX ; (11) 6982 5221. email: stiefano@uol.com.br

Recebido para publicação em 31/7/03. Aprovado, após revisão, em 16/10/03. 


\section{INTRODUÇÃO}

A cirrose hepática é a principal causa de hipertensão portal e ascite, a qual por sua vez é a principal complicação encontrada em pacientes cirróticos após aproximadamente dez anos de diagnóstico de cirrose compensada, acometendo 50\% dos doentes ${ }^{(1-3)}$.

Várias alterações podem ser detectadas pelas provas de função pulmonar em doentes portadores de doença hepática crônica, principalmente naqueles com cirrose. Essas alterações, que em conjunto caracterizam a "síndrome hepatopulmonar", acarretam hipoxemia e ocorrem em um terço dos doentes portadores de cirrose ${ }^{(4)}$.

A oxigenação inadequada nos portadores de cirrose é causada por vários mecanismos fisiopatológicos, como tônus vascular inadequado, vasodilatação pulmonar, relação ventilação-perfusão alterada (V/Q), aumento de shunts arterio-venosos e alterações na relação difusão-perfusão ${ }^{(4-9)}$.

Além das alterações já esperadas nos cirróticos, quando ocorre ascite podemos observar alterações restritivas e obstrutivas nas provas de função pulmonar ${ }^{(10)}$, com diminuição dos volumes pulmonares e hipoxemia, que melhoram após redução da ascite ${ }^{(11-15)}$.

0 aumento do volume e da pressão intraabdominal decorrentes da ascite levam à diminuição da expansibilidade pulmonar, com conseqüente hipoventilação, principalmente nas bases pulmonares. Juntamente com o edema intersticial presente nos pacientes cirróticos, pode haver colapso alveolar e microatelectasias que podem explicar as alterações espirométricas e gasométricas encontradas ${ }^{(10)}$.

Portanto, a ascite, principalmente quando de grande volume, prejudica de forma variável a função pulmonar de doentes com hipertensão portal de diferentes etiologias, sobretudo quando associada à cirrose. Este trabalho objetiva avaliar e quantificar a influência da redução do volume do líquido ascítico na função pulmonar de doentes portadores de hipertensão portal.

\section{MÉTODO}

Analisamos a função pulmonar através de espirometria e medida dos gases arteriais, em doentes adultos, de ambos os sexos, com diagnóstico de hipertensão portal e ascite, internados no Hospital Central e no Hospital São Luiz Gonzaga, ambos pertencentes à Irmandade Santa Casa de Misericórdia de São Paulo (ISCMSP), no período de março de 1996 a outubro de 2000.

Todos os doentes foram admitidos no grupo a ser estudado, após serem devidamente esclarecidos em relação ao tratamento proposto e terem concordado com a realização dos exames espirométrico e gasometria arterial.

Os critérios de exclusão foram: doenças cardíacas e pulmonares prévias, instabilidade hemodinâmica, neoplasias, insuficiência renal com creatinina sérica $>3,5 \mathrm{mg} / \mathrm{dl}$, hemorragia gastrointestinal, encefalopatia, peritonite bacteriana e dificuldade em colaborar com a realização do exame espirométrico. Os doentes tabagistas foram incluídos apenas quando não apresentavam sintomas ou sinais clínicos ou radiológicos de comprometimento pulmonar prévio.

Os radiografias torácicos mostraram-se dentro dos limites da normalidade, apenas com diminiução da expansibilidade pulmonar como conseqüência do grande volume abdominal apresentado pelos pacientes analisados.

Os doentes cirróticos incluídos foram classificados de acordo com os critérios de Child-Pugh para estabelecimento da gravidade da doença (Quadro 1).

Todos os doentes apresentavam-se com ascite de quantidade moderada a intensa, o que foi caracterizado pelos dados do exame

\begin{tabular}{|c|c|c|c|}
\hline \multicolumn{4}{|c|}{$\begin{array}{c}\text { QUADRO } 1 \\
\text { Classificação de Child - Pugh }\end{array}$} \\
\hline Pontuação & 1 & 2 & 3 \\
\hline Encefalopatia & Nenhuma & $1-2$ & $3-4$ \\
\hline Ascite & Ausente & Leve & Moderada \\
\hline Bilirrubina & $1-2$ & $2-3$ & $>3$ \\
\hline Albumina & $>3,5$ & $2,8-3,5$ & $<2,8$ \\
\hline TP (Seg. prolongados) & $1-4$ & $4-6$ & $>6$ \\
\hline
\end{tabular}


físico e ultrassonográficos: ascite de grande volume, pela simples inspeção do abdome; ascite com aumento da tensão da parede abdominal, constatada pela palpação; ascite que pela sua proporção causava desconforto respiratório ao doente, em especial quando em decúbito dorsal; e ultra-som evidenciando ascite volumosa.

Todos os doentes submeteram-se ao estudo da função pulmonar antes do início do tratamento da ascite. 0 tratamento clínico constituiu-se de prescrição de repouso, dieta com 2,0g de sal e diuréticos. 0 diurético utilizado foi a espironolactona, associada ou não à furosemida. Os pacientes não foram submetidos a tratamento fisioterápico pulmonar.

A paracentese terapêutica foi indicada nos doentes que apresentassem ascite com sinais e sintomas de desconforto abdominal e respiratório ou nos doentes que não estivessem respondendo satisfatoriamente ao tratamento clínico com diuréticos (perda contínua de peso de cerca de $500 \mathrm{~g} / \mathrm{dia}$ ).

Retirou-se uma média de 6,5 litros de líquido ascítico por doente, com reposição parenteral de uma unidade de plasma $(300 \mathrm{ml}$ ) para cada 2 litros de líquido ascítico drenado. Após a realização da paracentese os doentes eram submetidos a espirometria, realizada no dia seguinte ao procedimento.

Os exames espirométricos foram realizados no Laboratório de Provas de Função Pulmonar da 1SCMSP com os aparelhos: Med Graphics Breeze Cardiorespiratory Diagnostic Software Model 1070; Espirometro Koko - programa 2.15; Instrumentation Laboratory INC- PH/ Gas Analyzer - model 113.

A técnica utilizada para realização do exame e os parâmetros obtidos e analisados estão de acordo com o Consenso Brasileiro de Espirometria (rotina estabelecida pelo Laboratório de Provas de Função Pulmonar da Santa Casa de São Paulo).

Os parâmetros analisados foram: CVF (capacidade vital forçada); VEF1 (volume expiratório no primeiro segundo); FEF 25-75\% (fluxo expiratório forçado entre 25 e 75\% da CVF); VRE (volume de reserva expiratória) e relação VEF1 / CVF.

A curva da capacidade vital lenta (CVL) avaliou os volumes e capacidades pulmonares e os valores preditos adotados foram os de Crapo ${ }^{(16)}$.
A curva volume-tempo (espirometria forçada) foi realizada atendendo os critérios de aceitabilidade e reprodutibilidade de curvas preconizados pela American Thoracic Society (ATS, 1987-1995), sendo escolhida a melhor de três curvas aceitáveis, de oito realizadas. Os valores preditos foram os de Knudson ${ }^{(17)}$.

A curva fluxo-volume também foi submetida aos critérios de aceitabilidade e reprodutibilidade de curvas da American Thoracic Society (ATS, 1987-1995), sendo escolhida a melhor de três curvas aceitáveis, e sendo realizada curva envelope.

Os doentes submetidos à gasometria arterial foram analisados quanto a sua pressão arterial de oxigênio ( $\mathrm{PaO2}$ ), pressão arterial de dióxido de carbono (PaCO2) e saturação arterial de oxigênio ( $\mathrm{SaO} 2)$.

Os resultados das variáveis encontradas foram analisadas pelo teste t de Student para dados pareados. Foram consideradas diferenças estatisticamente significativas quando $p<0,050$ (5\%).

\section{RESULTADOS}

Analisamos 15 doentes portadores de hipertensão portal e ascite, sendo 9 (60\%) do sexo masculino, e 6 (40\%) do sexo feminino, com idade variável de 31 a 67 anos e média de $51 \pm 9,5$ anos. Observou-se maior prevalência de doentes com diagnóstico de cirrose hepática alcoólica (5 doentes - 33,33\%). A hipertensão portal associada ao vírus da hepatite B ou C, ou esquistossomose, teve prevalência de 60\% (9 doentes) (Tabela 1). Dez doentes tinham antecedentes de alcoolismo (67\%) e 5 doentes eram tabagistas (33,3\%).

TABELA 1

Distribuição segundo a freqüência dos diagnósticos etiológicos dos doentes analisados no periodo de mar/96 a out/00, na ISCMSP

\begin{tabular}{lcl}
\hline Diagnósticos & Número de doentes & $\mathbf{( \% )}$ \\
\hline Cirrose alcoólica & 5 & 33,33 \\
Esquistossomose ( EM ) & 2 & 13,33 \\
Cirrose alcoólica e EM & 1 & 6,66 \\
Cirrose alcoólica e vírus C & 2 & 13,33 \\
Cirrose alcoólica e vírus B & 1 & 6,33 \\
Cirrose por vírus B & 1 & 6,33 \\
Cirrose criptogênica & 2 & 13,33 \\
Trombose veia porta & 1 & 6,33 \\
\hline Total & 15 & 100 \\
\hline
\end{tabular}


Segundo os critérios de Child-Pugh, aplicados a 13 pacientes, excetuando-se os pacientes 1 e 3, portadores de esquistossomose, nosso grupo foi formado em sua maioria por doentes do escore B (11 doentes/84,6\%) sendo que apenas 2 doentes eram de escore $C$ $(15,4 \%)$ e nenhum doente era do escore A.

Doze doentes foram submetidos à paracentese terapêutica, com retirada de 6,5 litros

TABELA 2

Resultados espirométricos obtidos pré e pós paracentese e/ou tratamento clínico da ascite nos doentes analisados no periodo de mar/96 a out/00, na ISCMSP

\begin{tabular}{lll}
\hline Paciente & Pré-tratamento & Pós-tratamento \\
\hline 1 & DVOPV & DVOPV \\
2 & DVOPV & NL \\
3 & DVOPV & DVOPV \\
4 & NL & NL \\
10 & DVM & NL \\
11 & DVOM & DVOL \\
12 & DVOL & NL \\
13 & DVOL & NL \\
14 & DVOL & DVOL \\
15 & DVM & DVM \\
16 & DVR & NL \\
17 & DVM & DVOL \\
18 & DVR & NL \\
19 & DVOL & NL \\
20 & NL & NL \\
\hline
\end{tabular}

Fonte: Laboratório de Provas de Função Pulmonar da Santa Casa de São Paulo; NL: Normal; DVOL : distúrbio ventilatório obstrutivo leve; DVOM: distúrbio ventilatório obstrutivo moderado; DVOPV: distúrbio ventilatório obstrutivo de pequenas vias aéreas ; DVR: distúrbio ventilatório restritivo; DVRM: distúrbi ventilatório restritivo moderado; DVM: distúrbio ventilatório misto. de líquido ascítico e perda de $6,9 \mathrm{~kg}$ de peso em média. Três doentes apresentaram excelente resposta ao tratamento clínico, com diminuição importante do volume abdominal, com perda de $6,4 \mathrm{~kg}$ de peso em média, e foram então encaminhados para nova espirometria sem a realização da paracentese terapêutica.

Os diagnósticos espirométricos encontrados nos doentes analisados encontram-se na Tabela 2.

Nossos doentes, antes da paracentese e/ou tratamento clínico da ascite, apresentaram resultados da CVF inferiores às medidas médias previstas e obtiveram melhora significativa desse parâmetro após o tratamento. Os valores de VEF1 também encontravam-se reduzidos antes do tratamento da ascite e melhoraram após a paracentese e/ou tratamento clínico. A relação VEF1 / CVF não mostrou diferença estatística significante após tratamento. Os valores de FEF 25-75\% antes da paracentese e/ou tratamento clínico da ascite também mostravam-se diminuídos em relação à média prevista. Apesar de observarmos um aumento após o tratamento, este não foi estatisticamente significante. Podemos observar que ocorreu uma melhora do VRE após redução da ascite, com significância estatística. Os resultados encontram-se listados na Tabela 3. Nessa tabela demonstramos resumidamente todas as principais variáveis por nós analisadas e seu grau de significância para o estudo em questão.

TABELA 3

Comparação das variáveis espirométricas e dos gases arterias entre pré e pós paracentese e/ou tratamento clínico da ascite

\begin{tabular}{|c|c|c|c|}
\hline VARIÁVEL & MÉDIA e D.PADRÃO PRÉ & MÉDIA e D.PADRÃO PÓS & SIGNIFICÂNCIA (p) \\
\hline CVF (L) & $2,67 \pm 0,67$ & $3,13 \pm 0,83$ & ${ }^{*} 0,002$ \\
\hline CVF $(\%)$ & $82,40 \pm 17,00$ & $97,53 \pm 12,51$ & ${ }^{*} 0,001$ \\
\hline $\operatorname{VEF}_{1}(\mathrm{~L})$ & $2,10 \pm 0,53$ & $2,45 \pm 0,61$ & ${ }^{*} 0,001$ \\
\hline $\mathrm{VEF}_{1}(\%)$ & $78,33 \pm 19,59$ & $93,80 \pm 16,74$ & ${ }^{*}<0,001$ \\
\hline $\mathrm{VEF}_{1} / \mathrm{CVF}(\%)$ & $78,07 \pm 10,02$ & $78,40 \pm 7,58$ & 0,727 \\
\hline FEF $25-75 \%$ (L/Seg) & $2,12 \pm 1,00$ & $2,35 \pm 0,85$ & 0,127 \\
\hline FEF $25-75 \%(\%)$ & $75,40 \pm 38,94$ & $83,87 \pm 33,43$ & 0,170 \\
\hline VRE (L) & $0,73 \pm 0,34$ & $1,0 \pm 0,50$ & ${ }^{*} 0,019$ \\
\hline VRE (\%) & $73,27 \pm 40,60$ & $100,00 \pm 37,42$ & ${ }^{*} 0,033$ \\
\hline $\mathrm{PaO}_{2}(\mathrm{mmHg})$ & $68,25 \pm 16,63$ & $75,84 \pm 17,01$ & ${ }^{*} 0,027$ \\
\hline $\mathrm{PaCO}_{2}(\mathrm{mmHg})$ & $31,95 \pm 7,89$ & $28,70 \pm 4,59$ & 0,217 \\
\hline $\mathrm{SaO}_{2}(\%)$ & $91,71 \pm 5,99$ & $94,32 \pm 4,18$ & ${ }^{*} 0,032$ \\
\hline
\end{tabular}

Teste aplicado: teste t de Student para resultados pareados; fonte: Laboratório de Provas de Função Pulmonar da Santa Casa de São Paulo; CVF: capacidade vital forçada; VEF1: volume expiratório forçado no primeiro segundo; FEF 25-75\% : fluxo expiratório forçado entre 25 e 75 \% da capacidade vital forçada; VRE: volume de reserva expiratória; Pa02: pressão arterial de oxigênio; PaC02: pressão arterial de gás carbônico; Sa02 : saturação arterial de oxigênio; *: significante. 
Seis doentes foram submetidos a gasometria arterial. Antes da paracentese os valores médios encontrados nesses doentes foram: $\mathrm{PaO} 2=68 \pm 17 \mathrm{mmHg} ; \mathrm{PaCO} 2=32 \pm 17 \mathrm{mmHg}$; e $\mathrm{SaO} 2=92 \pm 6 \%$. Após a paracentese, as médias foram: $\mathrm{PaO} 2=76 \pm 17 \mathrm{mmHg} ; \mathrm{PaCO} 2=$ $29 \pm 5 \mathrm{mmHg}$ e $\mathrm{SaO} 2=94 \pm 4 \%$. 0 incremento nos valores de $\mathrm{PaO} 2$ e $\mathrm{SaO} 2$ mostraram significância estatística (Tabela 3).

\section{DISCUSSÃO}

Mesmo com uma casuística pequena, observamos prevalência maior de doentes do sexo masculino, com diagnóstico de cirrose hepática alcoólica, em concordância com a literatura ${ }^{(1,2,18)}$.

Segundo vários trabalhos ${ }^{(4-9,19,20)}$, as alterações pulmonares encontradas nos doentes cirróticos estão intimamente relacionadas ao grau de comprometimento da função hepática. Esse dado é importante para a interpretação dos resultados do nosso trabalho, visto que nossos doentes eram na maioria cirróticos (60\%) com grau moderado a avançado de doença (ChildPugh B ou C em 86,66\% dos casos - 13 doentes), podendo apresentar manifestações pulmonares decorrentes da sua doença de base, além das ocasionadas pelo aumento da pressão intra-abdominal como conseqüência da ascite.

Observamos maior prevalência de distúrbio ventilatório obstrutivo (oito doentes - 53,32\%), sendo que na literatura os relatos apontam para uma maior prevalência de distúrbio ventilatório restritivo ${ }^{(10,14,21-23)}$.

Segundo Yao et al. ${ }^{(10)}$, a ascite acarreta elevação do diafragma, com diminuição dos volumes pulmonares e aumento da pressão intratorácica, que juntamente com o edema pulmonar, encontrado nos pacientes cirróticos, pode levar à compressão do tecido pulmonar, causando microatelectasias que seriam responsáveis pelo padrão restritivo encontrado nas provas de função pulmonar e pela hipoxemia. Como em nosso trabalho, Yao et al. ${ }^{(10)}$ relataram também a presença de distúrbio ventilatório obstrutivo e concluíram que esse achado poderia estar relacionado ao grau de comprometimento hepático dos doentes analisados. Nos doentes com doença hepática mais avançada haveria maior edema intersticial pulmonar e, portanto, maior envolvimento dos alvéolos e bronquíolos, causando fechamento precoce das vias aéreas na expiração e distúrbios obstrutivos.

Segundo Chao et al. ${ }^{(21)}$, os efeitos da ascite no sistema respiratório são mediados, provavelmente, pela pressão hidrostática exercida sobre o diafragma e a gravidade dos prejuízos causados nas trocas gasosas está intimamente relacionada à diminuição dos volumes pulmonares que ocorre nessas circunstâncias.

Não pudemos estabelecer relação entre tabagismo e distúrbio ventilatório obstrutivo, pois apenas três doentes, dos oito com padrão obstrutivo, eram tabagistas. Acreditamos, assim como Yao et al. ${ }^{(10)}$, que as alterações pulmonares decorrentes da hepatopatia crônica avançada podem por si justificar melhor nossos achados.

Ordialez Fernandez et al. ${ }^{(22)}$ observaram a presença de padrão restritivo, com diminuição do volume de reserva expiratória (VRE) na avaliação funcional pulmonar, em doentes cirróticos com e sem ascite. Encontraram diminuição nos parâmetros analisados ao medir a força muscular respiratória em doentes com e sem ascite, indicando uma menor efetividade dos músculos da caixa torácica, o que poderia contribuir para as alterações funcionais encontradas.

$\mathrm{Na}$ maioria dos trabalhos revistos observouse, como nós, diminuição da CVF, do VEF1, do VRE, além da CRF (capacidade residual funcional) antes, e aumentos significativos após a paracentese ${ }^{(10-12,14,15,21,24)}$.

Observamos que vários doentes apresentaram exame espirométrico normal após o tratamento clínico com diuréticos, ou após a paracentese, evidenciando melhora evidente dos parâmetros respiratórios pela diminuição do volume da ascite.

$A$ relacão VEF1/CVF não apresentou diferenças significativas antes e após o tratamento, o que mostra que os aumentos na VEF1 foram proporcionais aos aumentos da CVF, ficando muito próximos dos $80 \%$ esperados para a relação. A diminuição do VEF1 e da CVF com manutenção dos valores preditos para a relação VEF1/CVF é encontrada nos distúrbios restritivos pulmonares, que podem estar, portanto, associados aos distúrbios obstrutivos observados.

A pequena diminuição no FEF25-75\% 
encontrada antes do tratamento pode estar associada a comprometimento bronquiolar e compressão pulmonar com fechamento precoce das pequenas vias aéreas, que pode ocorrer em doentes hepatopatas com ascite.

0 VRE mostrou-se bem diminuído em nosso estudo antes do tratamento da ascite com melhora significativa após seu tratamento, em concordância com os trabalhos revistos. Acreditamos, como outros autores, que a diminuição da pressão intra-abdominal pela diminuição do volume de ascite, foi a responsável pela melhora no VRE ${ }^{(11,14,22)}$.

Segundo Chao et al. ${ }^{(21)}$, a ascite pode comprometer a perfusão pulmonar pela diminuição do fluxo sangüíneo nas áreas basais dos pulmões, que se encontram comprimidas mecanicamente, sendo um fator agravante de hipoxemia nos pacientes cirróticos. Alguns trabalhos encontraram hipoxemia nos doentes ascíticos antes do tratamento e relataram aumento importante na $\mathrm{Pa} 02$ após a terapia com diuréticos ${ }^{(15,23)}$. Possivelmente o uso de diuréticos, segundo os autores, diminuiu o edema intersticial pulmonar, acarretando uma relação ventilação/perfusão mais favorável.

No presente estudo, encontramos hipoxemia leve em nossos doentes com melhora após 0 tratamento. A $\mathrm{SaO}^{2}$ também melhorou após a redução da ascite mas não observamos o mesmo quando avaliamos a $\mathrm{PaCO}^{2}$. Provavelmente a melhora nos volumes pulmonares, com conseqüente melhora na ventilação pulmonar, contribuiu para a melhor oxigenação. Como todos os doentes fizeram uso de diuréticos durante o estudo, em concordância com os autores citados, isto pode ter contribuído para uma melhora na $\mathrm{PaO}^{2}$.

Em resumo, nos casos estudados, a diminuição do volume da ascite melhora significativamente a ventilação pulmonar, sendo que a paracentese terapêutica parece ser uma alternativa de tratamento para um alívio rápido dos sintomas de dispnéia e desconforto abdominal, ou para os casos em que a terapia com diuréticos não esteja sendo totalmente satisfatória.

\section{Referências:}

1. Runyon BA. Care of patients with ascites. N Engl J Med 1994;330:337-42.

2. Jaffe DL, Chung RT, Friedman LS. Management of portal hypertension and its complications. Med Clin North Am 1996;80:1021-34

3. Forouzandeh B, Konicek F, Sheagren JN. Large-volume paracentesis in the treatment of cirrhotic patients with refractory ascites. The role of postparacentesis plasma volume expansion. J Clin Gastroenterol 1996;22:207-10.

4. Rodríguez-Roisin R, Agustí AGN, Roca J. The hepatopulmonary syndrome: new name, old complexities. Thorax 1992;47:897-902.

5. Rodríguez-Roisin R, Roca J, Agustí AGN, Mastai R, Wagner PD, Bosh J. Gas exchange and pulmonary reactivity in patients with liver cirrhosis. Am Rev Respir Dis1987;135:1085-92.

6. Edell ES, Cortese DA, Krowka MJ, Rehder K. Severe hypoxemia and liver disease. Am Rev Respir Dis 1989;140:1631-5.

7. Mélot C, Naeije R, Dechamps P, Hallemans R, Lejeune P. Pulmonary and extrapulmonary contributors to hypoxemia in liver cirrhosis. Am Rev Respir Dis 1989;139:632-40.

8. Kao CH,Huang CK, Tsai SC, Wang SJ, Chen GH. Evaluation of lung ventilation and permeability in cirrhosis. J Nucl Med1996;37:437-41.

9.King PD, Rumbaut R, Sanchez C. Pulmonary manifestations of chronic liver disease. Dig Dis 1996;14:73-82.

10. Yao EH, Kong BC, Hsue GL, Zhou AC, Wang GH. Pulmonary function changes in cirrhosis of the liver. $\mathrm{Am} \mathrm{J}$ Gastroentero 1987;82:352-4.

11.Berkowitz KA, Butensky MS, Smith RL. Pulmonary function changes after large volume paracentesis. Am J Gastroenterol 1993;88:905-7.

12.Nagral A, Kolhatkar VP, Bhatia SJ, Taskar VS, Abraham P. Pulmonary function tests in cirrhotic and non cirrhotic portal hypertension. Indian J Gastroenterol 1993;12:36:40. [abstract].

13.Angueira CE, Kadakia S. Effects of large-volume paracentesis on pulmonary function in patients with tense cirrhotic ascites. Hepatology 1994;20:825-8.

14.Byrd RP, Roy TM, Simons M. Improvement in oxygenation after volume paracentesis. South Med J 1996;89:689-92.

15. Chang SC, Chang Hl, Chen FJ, Shiao GM, Wang SS, Lee SD. Therapeutic effects of diuretics and paracentesis on lung function in patients with non-alcoholic cirrhosis and tense ascites. J Hepatol 1997;26:833-8.

16.Crapo RO, Morris AH, Clayton PD, Nixon CR. Lung volumes in healthy nonsmoking adults. Bull Eur Physiopathol Respir 1982;18:419-25.

17.Knudson RJ, Lebowitz MD, Holberg CJ, Burrows B. Changes in the normal maximal expiratory flow-volume curve with growth and aging. Am Rev Respir Dis 1983;127:725-34.

18.Strauss E. Ascite: tratamento clínico e complicações. HFA Pub Téc Cient 1991;6:38-40.

19.Agusti AGN, Roca J, Bosh J, Rodriguez-Roisin R. The lung in patients with cirrhosis. J Hepatol 1990;10:251-7.

20.Robert V, Chabot F, Vial B, Guiot P, Poussel JF, Polu JM Hepatopulmonary syndrome: physiopathology of impaired gas exchange. Rev Mal Respir 1999;16:769-79.

21.Chao Y, Wang SS, Lee SD, Shiao GM, Chang HI, Chang SC. Effect of large-volume paracentesis on pulmonary function in patients with cirrhosis and tense ascites. J Hepatol 1994;20:101-5.

22.Ordiales Fernandez JJ, Fernandez Moya A, Nistal de Paz F, Linares Rodrigues A, Colubi Colubi L, Alvarez Asensio E, et at. Influence of liver cirrhosis with and without ascites on ventilatory mechanics. Rev Esp Enferm Dig 1995;87:853-7.

23.Gupta D, Lalrothuama, Agrawal PN, Aggarwal NA, Dhiman RK, Behera D, Chawla Y. Pulmonary function changes after large volume paracentesis. Trop Gastroenterol 2000;21:68-70. 